Case Report

\title{
Rhabdomyolysis in a Hospitalized 16-Year-Old Boy: A Rarely Reported Underlying Cause
}

\author{
Rishika Singh, ${ }^{1}$ Dilip R. Patel, ${ }^{1}$ and Sherry Pejka ${ }^{2}$ \\ ${ }^{1}$ Department of Pediatric and Adolescent Medicine, Western Michigan University Homer Stryker MD School of Medicine, \\ Kalamazoo, MI, USA \\ ${ }^{2}$ Bronson Children's Hospital, Kalamazoo, MI, USA
}

Correspondence should be addressed to Dilip R. Patel; dilip.patel@med.wmich.edu

Received 13 July 2016; Accepted 20 October 2016

Academic Editor: Gulay Karagüzel

Copyright (C) 2016 Rishika Singh et al. This is an open access article distributed under the Creative Commons Attribution License, which permits unrestricted use, distribution, and reproduction in any medium, provided the original work is properly cited.

Rhabdomyolysis can occur because of multiple causes and account for 7\% of all cases of acute kidney injury annually in the United States. Identification of specific cause can be difficult in many cases where multiple factors could potentially cause rhabdomyolysis. We present a case of 16-year-old male who had seizures and was given levetiracetam that resulted in rhabdomyolysis. This side effect has been rarely reported previously and like in our case diagnosis may be delayed.

\section{Case Report}

A 16-year-old, previously healthy, well-built male presented to the hospital after having two witnessed generalized tonic clonic seizures. The first seizure occurred at school and lasted for 2 minutes. The second seizure occurred in the emergency department and lasted for 2.5 minutes. The patient was given lorazepam and intravenous levetiracetam. He was conscious and aware of his surroundings between the two seizures and 1 minute after the second seizure. There was no reported fall or other trauma to the patient before or during the seizure. Initial labs revealed serum bicarbonate of $18 \mathrm{mmol} / \mathrm{L}$, normal serum electrolytes, and creatinine. Other negative studies included urinalysis, urine drug screen (screened for opiates, barbiturates, phencyclidine, amphetamines, cocaine, cannabinoids, and benzodiazepines), ethanol, acetaminophen, and salicylate levels in the blood. Patient was given a normal saline bolus followed by maintenance fluid. In consultation with neurology, levetiracetam level was not obtained.

Levetiracetam was continued at $750 \mathrm{mg}$ orally every 12 hours. The following day, patient developed back pain. It was located lateral to lumbosacral spine on both sides and the area was tender to palpation. Creatine kinase (CK) drawn at this time was $565 \mathrm{U} / \mathrm{L}$; creatinine $(\mathrm{Cr})$ was elevated at $2.2 \mathrm{mg} / \mathrm{dL}$. Repeat urinalysis was performed and was positive for myoglobin, negative for protein, red blood cells, white blood cells, and bacteria. At this point, it was thought that rhabdomyolysis was secondary to the seizure and the treatment was continued. We omitted potassium from the fluids and increased the rate to $200 \mathrm{~mL} / \mathrm{hr}$ to provide more aggressive hydration. We continued to check creatine kinase and renal function every 6 hours. Patient's back pain continued to worsen. The pain was now also involving his upper back and thighs. He was requiring narcotics for pain control. Creatine kinase continued to rise exponentially and was $15,111 \mathrm{U} / \mathrm{L}$ on day 4 ; creatinine remained elevated with only mild fluctuations. We discontinued levetiracetam and switched to devalproex sodium on day 5 . This was followed by improvement in patient's back pain the next day.

Patient's back pain completely resolved in 2 days. There was a steady and quick decline in the creatine kinase level and creatinine. Creatinine normalized by day 7 and creatine kinase by day 10. Patient was discharged home on devalproex sodium. He has been doing well since discharge. Creatine kinase levels have been checked multiple times following discharge and have remained normal. Subsequent renal function tests have also remained normal. 


\section{Discussion}

In this patient our diagnosis was rhabdomyolysis and subsequent acute kidney injury (AKI) due to levetiracetam. Rhabdomyolysis is a common clinical syndrome that results from the rapid breakdown of skeletal muscle fibers, which leads to leakage of potentially toxic cellular contents into the systemic circulation [1]. It ranges from an asymptomatic illness with elevation of creatine kinase level to a lifethreatening condition associated with extreme elevations in creatine kinase, electrolyte imbalances, acute renal failure, and disseminated intravascular coagulation [2]. The classic reported triad of symptoms includes muscle pain, weakness, and dark urine, although more than $50 \%$ of the patients do not complain of muscle pain or weakness and the classic triad is seen in $<10 \%$ patients [3]. Nonspecific symptoms like fever, malaise, nausea, and vomiting may be present [2].

More than 25,000 cases are reported annually in the USA, which account for $7 \%$ of all cases of AKI [4]. Acute renal failure from rhabdomyolysis in children is much less common $(\sim 5 \%)$ than the risk reported for adults [5]. Theoretically, any form of muscle damage can lead to rhabdomyolysis. In the pediatric population, viral myositis, trauma, connective tissue disorders, exercise, and drug overdose are responsible for much of the rhabdomyolysis seen in these patients $[2,5]$. There are close to 200 medications and toxins known to cause skeletal muscle injury [4,6]. In some cases a cause of rhabdomyolysis cannot be clearly established [7].

Diagnosis is based on the elevation of creatine kinase $>10$ times the normal value. It rises within 2-12 hours of muscle injury, peaks around 24-72 hours, and then declines over the next 3-5 days [1,8]. Myoglobinuria can be present which may be discovered as positive urine dipstick test for blood in absence of red blood cells. Other electrolyte derangements may be present including hyperkalemia, hyperphosphatemia, hypocalcemia, and lactic acidosis [4]. Renal function should be assessed since there is a risk of AKI [1]. Electrocardiogram should be obtained if electrolyte abnormalities are present. Other complications that can occur in patients with rhabdomyolysis are compartment syndrome and disseminated intravascular coagulation [2].

The main objective in management is to prevent or treat AKI which is achieved mainly by aggressive hydration [2]. Due to accumulation of water in the muscle fibres and subsequent hypovolemia, fluid resuscitation is imperative to prevent prerenal azotemia [4]. Normal saline should be used without potassium or lactate. Intravenous infusion should be given at $1.5 \mathrm{~L} / \mathrm{hr}$ to maintain urine output of $200 \mathrm{~mL} / \mathrm{hr}$ and continued until creatine kinase value has declined to $<1000 \mathrm{IU} / \mathrm{L}[3,9]$. If the offending agent is suspected to be a medication, it should be discontinued promptly [4]. Evidence supporting the use of mannitol and/or bicarbonate comes mostly from animal studies and is inconsistent and conflicting. Recommendations for the use of bicarbonate to alkalinize the urine are based on retrospective studies in adults with no supporting data in the literature on pediatric patients [10]. Renal function and electrolytes should be monitored closely. Some patients with severe AKI may need hemodialysis. Prompt fasciotomy is required for patients with compartment syndrome [4].

Levetiracetam is generally considered safe and a commonly used antiseizure medication in pediatric population. Rhabdomyolysis is not a well-documented or listed adverse effect on this drug's profile but rare case reports have implicated levetiracetam as a cause of rhabdomyolysis [11, 12].

Based on this previously reported side effect of levetiracetam and temporal relationship between clinical and lab improvement with discontinuation of the drug, we surmise that rhabdomyolysis in our patient was due to this drug. In patients with elevated creatine kinase, drug induced rhabdomyolysis should always be considered.

\section{Competing Interests}

The authors declare that there is no conflict of interests regarding the publication of this paper.

\section{References}

[1] R. Zutt, A. J. van der Kooi, G. E. Linthorst, R. J. A. Wanders, and M. de Visser, "Rhabdomyolysis: review of the literature," Neuromuscular Disorders, vol. 24, no. 8, pp. 651-659, 2014.

[2] P. A. Torres, J. A. Helmstetter, A. M. Kaye, and A. D. Kaye, "Rhabdomyolysis: pathogenesis, diagnosis and treatment," The Ochsner Journal, vol. 15, no. 1, pp. 58-69, 2015.

[3] E. F. Elsayed and R. F. Reilly, "Rhabdomyolysis: a review, with emphasis on the pediatric population," Pediatric Nephrology, vol. 25, no. 1, pp. 7-18, 2010.

[4] R. Mannix, M. L. Tan, R. Wright, and M. Baskin, "Acute pediatric rhabdomyolysis: causes and rates of renal failure," Pediatrics, vol. 118, no. 5, pp. 2119-2125, 2006.

[5] G. Cervellin, I. Comelli, and G. Lippi, "Rhabdomyolysis: historical background, clinical, diagnostic and therapeutic features," Clinical Chemistry and Laboratory Medicine, vol. 48, no. 6, pp. 749-756, 2010.

[6] T. J. Coco and A. E. Klasner, "Drug-induced rhabdomyolysis," Current Opinion in Pediatrics, vol. 16, no. 2, pp. 206-210, 2004.

[7] G. Melli, V. Chaudhry, and D. R. Cornblath, "Rhabdomyolysis: an evaluation of 475 hospitalized patients," Medicine, vol. 84, no. 6, pp. 377-385, 2005.

[8] M. L. Shapiro, A. Baldea, and F. A. Luchette, "Rhabdomyolysis in the intensive care unit," Journal of Intensive Care Medicine, vol. 27, no. 6, pp. 335-342, 2012.

[9] S. G. Holt and K. P. Moore, "Pathogenesis and treatment of renal dysfunction in rhabdomyolysis," Intensive Care Medicine, vol. 27, no. 5, pp. 803-811, 2001.

[10] J. F. Eneas, P. Y. Schoenfeld, and M. H. Humphreys, “The effect of infusion of mannitol-sodium bicarbonate on the clinical course of myoglobinuria," Archives of Internal Medicine, vol. 139, no. 7, pp. 801-805, 1979.

[11] H. Akiyama, Y. Haga, N. Sasaki, T. Yanagisawa, and Y. Hasegawa, "A case of rhabdomyolysis in which levetiracetam was suspected as the cause," Epilepsy and Behavior Case Reports, vol. 2, pp. 152-155, 2014.

[12] J. E. Isaacson, D. J. Choe, and M. J. Doherty, "Creatine phosphokinase elevation exacerbated by levetiracetam therapy," Epilepsy and Behavior Case Reports, vol. 2, pp. 189-191, 2014. 


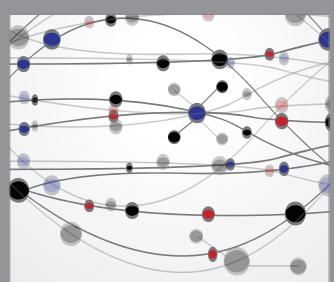

The Scientific World Journal
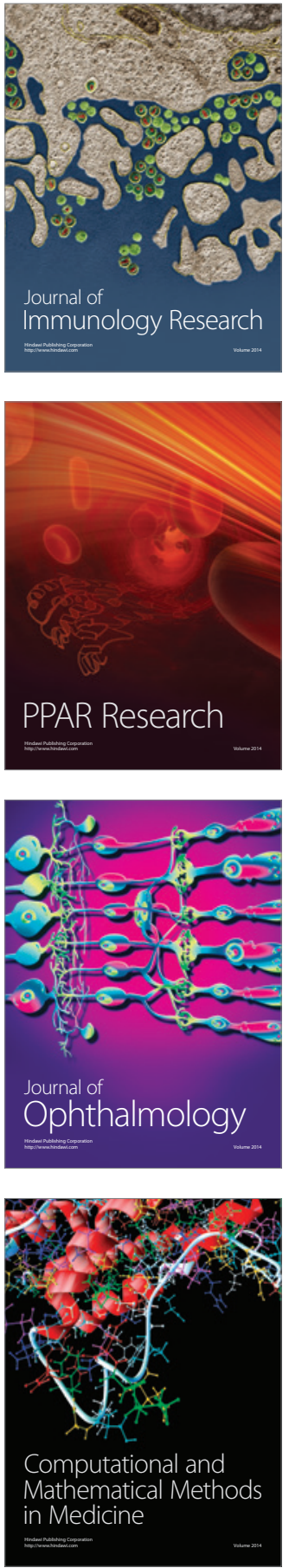

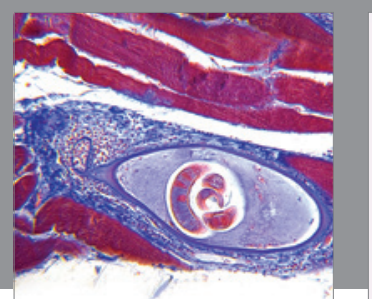

Gastroenterology Research and Practice

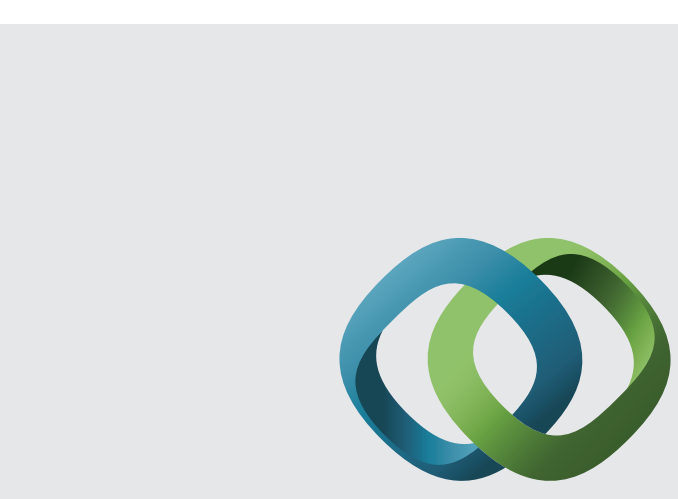

\section{Hindawi}

Submit your manuscripts at

http://www.hindawi.com
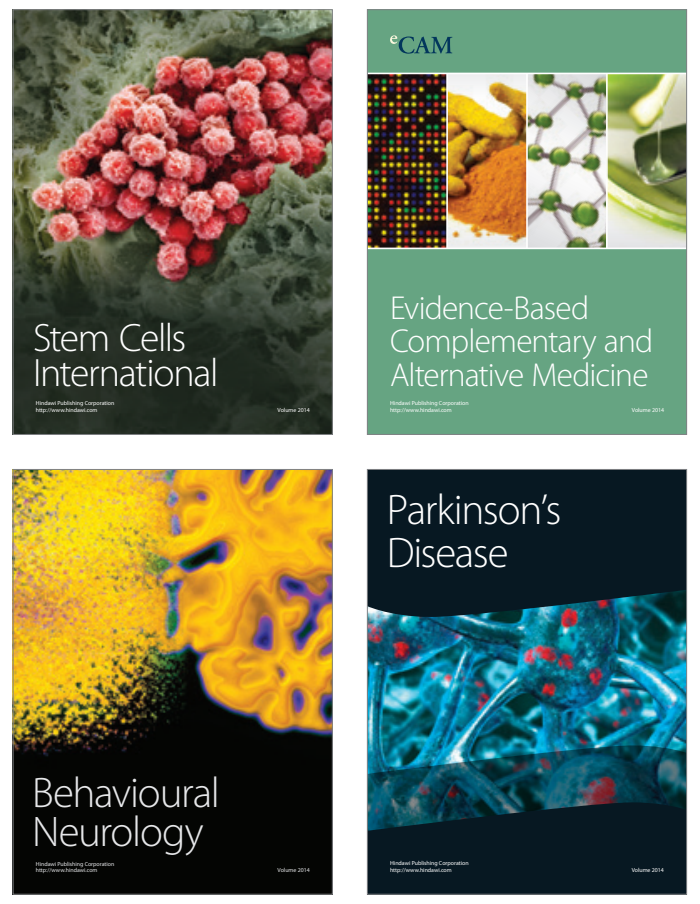
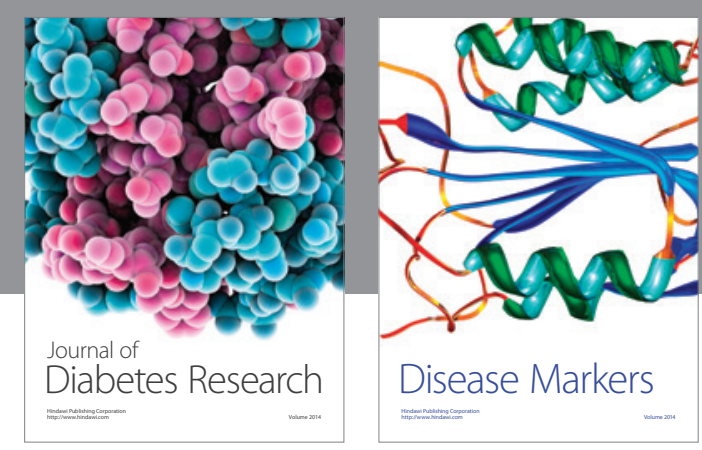

Disease Markers
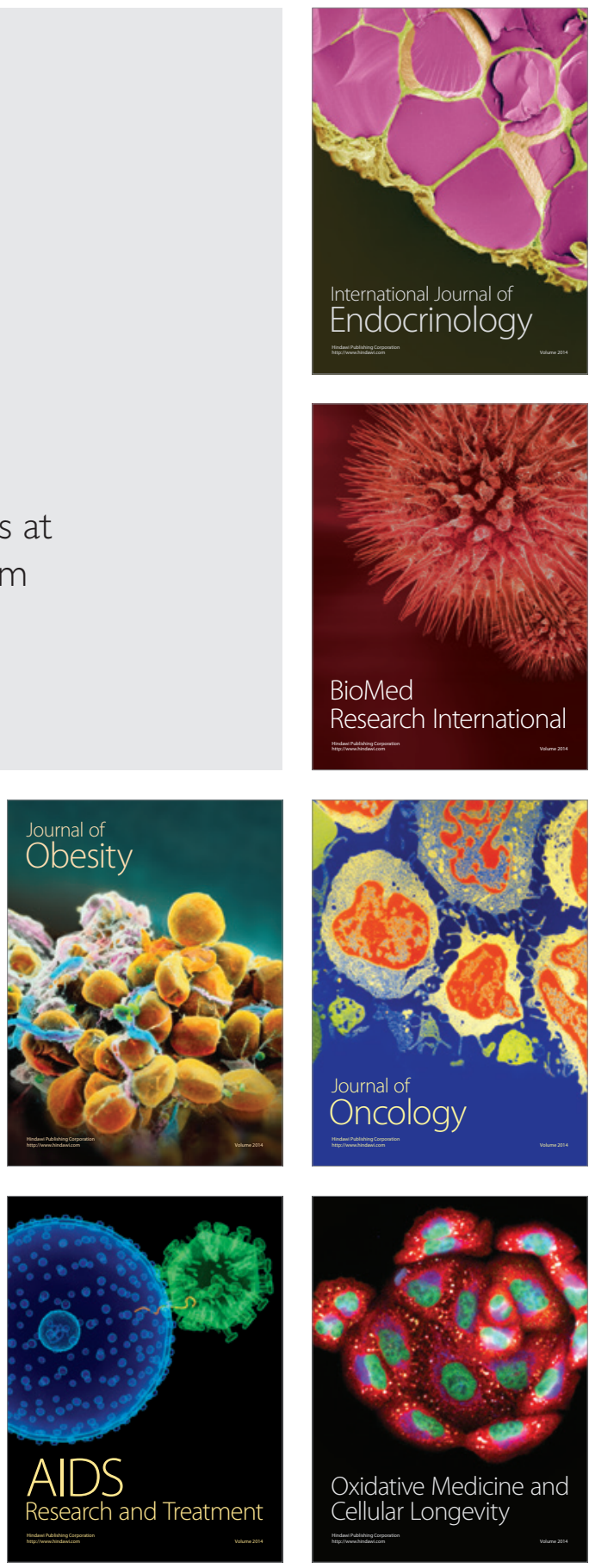